Supporting Information Available

\title{
Synthesis of very short chain Lysophosphatidyloligodeoxynucleotides
}

\author{
Rosa Chillemi, Danilo Aleo, Giuseppe Granata and Sebastiano Sciuto*
}

Dipartimento di Scienze Chimiche, Università di Catania, viale A. Doria 6, 95125, Catania, Italy.

\section{Table of contents}

Page 2: ${ }^{1} \mathrm{H}$ NMR (500 MHz) and ${ }^{13} \mathrm{C}$ NMR (125.7 MHz) spectra of 5'-GPd(ApApApT) (4).

Page 4: ${ }^{1} \mathrm{H}$ NMR (500 MHz) and ${ }^{13} \mathrm{C}$ NMR (125.7 MHz) spectra of 5'-GPd(ApApApApT) (5).

Page 6: ${ }^{1} \mathrm{H}$ NMR (500 MHz) spectrum of 5'-GPd(ApTpApTpTpA) (6).

Page 7: ${ }^{1} \mathrm{H}$ NMR (500 MHz) spectrum of 5'-GPd(GpA) (7).

Page 8: ${ }^{1} \mathrm{H}$ NMR (500 MHz) spectrum of 5'-GPd(TpA) (8).

Page 9: ${ }^{1} \mathrm{H}$ NMR (500 MHz) and ${ }^{13} \mathrm{C}$ NMR (125.7 MHz) spectra of 1-O-Benzoyl-3-O-(4,4'-dimethoxytrityl)$s n$-glycerol (16). 
[5'-GPd(ApApApT) (4)]. ${ }^{1} \mathrm{H}$ NMR $\left(\mathrm{D}_{2} \mathrm{O}\right): \delta 1.71\left(\mathrm{~d}, \mathrm{~J}=1.0 \mathrm{~Hz}, 3 \mathrm{H}, \mathrm{CH}_{3} \mathrm{~T} 4\right), 2.24$ (ddd partially overlapping with next signal, $\mathrm{J}_{2^{\prime}, 2^{\prime}}=-14.1, \mathrm{~J}_{2^{\prime}, 1^{\prime}}=7.0, \mathrm{~J}_{2^{\prime}, 3^{\prime}}=6.6 \mathrm{~Hz}, 1 \mathrm{H}, 2^{\prime}-\mathrm{H}$ T4), 2.27 (ddd partially overlapping with previous signal, $\mathrm{J}_{2^{\prime \prime}, 2^{\prime}}=-14.1, \mathrm{~J}_{2^{\prime}, 1^{\prime}}=6.7, \mathrm{~J}_{2^{\prime}, 3^{\prime}}=4.5 \mathrm{~Hz}, 1 \mathrm{H}, 2^{\prime \prime}-\mathrm{H}$ T4), 2.53 (ddd partially overlapping with next signal, $\mathrm{J}_{2^{\prime}, 2^{\prime \prime}}=-14.0, \mathrm{~J}_{2^{\prime}, 1^{\prime}}=9.2, \mathrm{~J}_{2^{\prime}, 3^{\prime}}=5.0 \mathrm{~Hz}, 1 \mathrm{H}, 2^{\prime}-\mathrm{H}$ A1), 2.57 (ddd partially overlapping with previous signal, $\mathbf{J}_{2^{\prime}, 2^{\prime \prime}}=-14.0, \mathrm{~J}_{2^{\prime}, 1^{\prime}}=9.8, \mathbf{J}_{2^{\prime}, 3^{\prime}}=5.6 \mathrm{~Hz}, 1 \mathrm{H}, 2^{\prime}-\mathrm{H}$ A2), 2.68-2.74 (overlapped multiplets, $3 \mathrm{H}, 2$ '"-H A1 and A2, 2'-H A3), 2.77 (ddd, $\mathrm{J}_{2^{\prime \prime}, 2^{\prime}}=-14.1, \mathrm{~J}_{2^{\prime}, 1^{\prime}}=6.0, \mathrm{~J}_{2^{\prime \prime}, 3^{\prime}}=3.0 \mathrm{~Hz}, 1 \mathrm{H}, 2^{\prime \prime}-\mathrm{H} \mathrm{A} 3$ ), $3.47\left(\mathrm{dd}, \mathrm{J}_{1 \mathrm{a}, 1 \mathrm{~b}}=-11.8, \mathrm{~J}_{1 \mathrm{a}, 2}=6.2 \mathrm{~Hz}, 1 \mathrm{H}, 1 \mathrm{a}-\mathrm{H}\right.$ of glycerol), $3.56\left(\mathrm{dd}, \mathrm{J}_{1 \mathrm{~b}, 1 \mathrm{a}}=-11.8, \mathrm{~J}_{1 \mathrm{~b}, 2}=4.2 \mathrm{~Hz}, 1 \mathrm{H}, 1 \mathrm{~b}-\mathrm{H}\right.$ of glycerol), 3.69 (ddd, $\mathrm{J}_{3 \mathrm{a}, 3 \mathrm{~b}}=-12.1, \mathrm{~J}_{3 \mathrm{a}, 2}=7.2, \mathrm{~J}_{3 \mathrm{a}, \mathrm{P}}=6.0 \mathrm{~Hz}, 1 \mathrm{H}, 3 \mathrm{a}-\mathrm{H}$ of glycerol), 3.77-3.82 (overlapped multiplets, $2 \mathrm{H}, 2-\mathrm{H}$ and $3 \mathrm{~b}-\mathrm{H}$ of glycerol), 3.95 (ddd, $\mathrm{J}_{5^{\prime}, 5^{\prime}}=-11.5, \mathrm{~J}_{5^{\prime \prime}, 4^{\prime}}=2.7, \mathrm{~J}_{5^{\prime \prime}, \mathrm{P}}=4.2 \mathrm{~Hz}, 1 \mathrm{H}, 5^{\prime \prime}-\mathrm{H} \mathrm{A1}$ ), $4.04\left(\mathrm{dt}, \mathrm{J}_{5^{\prime}, 5^{\prime}}=-11.5, \mathrm{~J}_{5^{\prime}, 4^{\prime}}=4.0, \mathrm{~J}_{5^{\prime}, \mathrm{P}}=4.0 \mathrm{~Hz}, 1 \mathrm{H}, 5^{\prime}-\mathrm{H}\right.$ A1), $4.09-4.29$ (partially overlapped multiplets, 7 H, 4'-H T4, 5'-H and 5"'-H T4, 5'-H and 5"'-H A2, 5'-H and 5'-H A3), 4.36 (m, 1 H, 4'-H A1), 4.42 (m, 1 H, 4'-H A2), 4.45 (m, 1 H, 4'-H A3), 4.57 (ddd, $\mathrm{J}_{3^{\prime}, 2^{\prime}}=6.6, \mathrm{~J}_{3^{\prime}, 2^{\prime}}=4.5, \mathrm{~J}_{3^{\prime}, 4^{\prime}}=4.0 \mathrm{~Hz}, 1 \mathrm{H}, 3^{\prime}-\mathrm{H}$ T4), 4.97 (m, $1 \mathrm{H}$, 3'-H A1), $5.01-5.04$ (overlapped multiplets, $2 \mathrm{H}, 3^{\prime}-\mathrm{H} \mathrm{A}^{\prime}$ and A3), 5.91 (dd, $\mathrm{J}_{1^{\prime}, 2^{\prime}}=9.8, \mathrm{~J}_{1^{\prime}, 2^{\prime \prime}}=5.2 \mathrm{~Hz}, 1 \mathrm{H}$, $\left.1^{\prime}-\mathrm{H} \mathrm{A} 2\right), 6.06\left(\mathrm{dd}, \mathrm{J}_{1^{\prime}, 2^{\prime}}=9.2, \mathrm{~J}_{1^{\prime}, 2^{\prime}}=5.6 \mathrm{~Hz}, 1 \mathrm{H}, 1^{\prime}-\mathrm{H} \mathrm{A1}\right), 6.11\left(\mathrm{dd}, \mathrm{J}_{1^{\prime}, 2^{\prime}}=7.0, \mathrm{~J}_{1^{\prime}, 2^{\prime}}=6.7 \mathrm{~Hz}, 1 \mathrm{H}, 1^{\prime}-\mathrm{H} \mathrm{T} 4\right)$, $6.20\left(\mathrm{dd}, \mathrm{J}_{1^{\prime}, 2^{\prime}}=8.0, \mathrm{~J}_{1^{\prime}, 2^{\prime}}=6.0 \mathrm{~Hz}, 1 \mathrm{H}, 1^{\prime}-\mathrm{H} \mathrm{A} 3\right), 7.43(\mathrm{q}, \mathrm{J}=1.0 \mathrm{~Hz}, 1 \mathrm{H}, 6-\mathrm{H} \mathrm{T} 4), 7.75(\mathrm{~s}, 1 \mathrm{H}, 2-\mathrm{H} \mathrm{A} 2)$, 7.88 (s, 1 H, 2-H A1), 8.00 (s, 1 H, 2-H A3), 8.19 (s, 1 H, 8-H A1), 8.20 (s, 1 H, 8-H A2), 8.31 (s, 1 H, 8-H A3) ppm.

${ }^{13} \mathrm{C}_{\mathrm{NMR}}\left(\mathrm{D}_{2} \mathrm{O}\right): \delta 14.3\left(\mathrm{CH}_{3} \mathrm{~T}\right.$ T), 39.8 (C-2' A1), 40.0 (C-2' A2), 40.8 (C-2' A3), 41.6 (C-2' T4), 64.9 (C-1

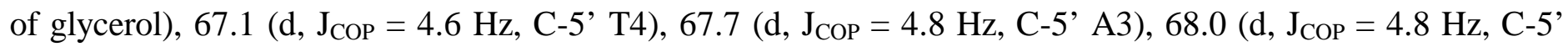
A2), $68.2\left(\mathrm{~d}, \mathrm{~J}_{\mathrm{COP}}=5.0 \mathrm{~Hz}, \mathrm{C}-5^{\prime}\right.$ A1), $69.1\left(\mathrm{~d}, \mathrm{~J}_{\mathrm{COP}}=5.0 \mathrm{~Hz}, \mathrm{C}-3\right.$ of glycerol), $72.4\left(\mathrm{C}-3^{\prime}\right.$ ' T4), $73.5\left(\mathrm{~d}, \mathrm{~J}_{\mathrm{CCOP}}=\right.$ $7.5 \mathrm{~Hz}, \mathrm{C}-2$ of glycerol), 78.1 (d, J $\mathrm{J}_{\mathrm{COP}}=5.0 \mathrm{~Hz}, \mathrm{C}-3^{\prime}$ A3), $79.2\left(\mathrm{~d}, \mathrm{~J}_{\mathrm{COP}}=5.0 \mathrm{~Hz}, \mathrm{C}-3^{\prime}\right.$ A2), 80.0 (d, J $\mathrm{J}_{\mathrm{COP}}=5.0$ Hz, C-3' A1), 84.9 (C-1' A2), 85.2 (C-1' A3), 86.1 (C-1' A1), 86.9 (C-1' T4), 87.2 (t, J CCOP $_{\text {( }}=9.8$ Hz, C-4'

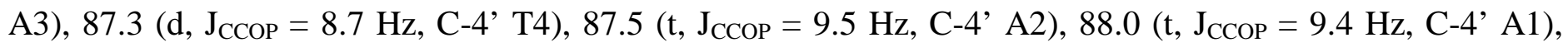
114.1 (C-5 T4), 120.7, 120.9 and 121.1 (C-5 A1, A2 and A3), 139.5 (C-6 T4), 141.8 (C-8 A3), 142.2 and 142.3 
(C-8 A1 and A2), 150.3, 150.5 and 150.9 (C-4 A1, A2 and A3), 153.8 (C-2 T4), 154.7 (C-2 A2), 154.9 (C-2 A1), 155.0 (C-2 A3), 157.1, 157.4 and 157.7 (C-6 A1, A2 and A3), 168.4 (C-4 T4) ppm. 
[5'-GPd(ApApApApT) (5)]. ${ }^{1} \mathrm{H}$ NMR $\left(\mathrm{D}_{2} \mathrm{O}\right): \delta 1.72\left(\mathrm{~d}, \mathrm{~J} \sim 0.9 \mathrm{~Hz}, 3 \mathrm{H}, \mathrm{CH}_{3}\right.$ T5), 2.24 (ddd partially overlapping with next signal, $\mathrm{J}_{2^{\prime}, 2^{\prime}}=-14.1, \mathrm{~J}_{2^{\prime}, 1^{\prime}}=6.9, \mathrm{~J}_{2^{\prime}, 3^{\prime}}=6.8 \mathrm{~Hz}, 1 \mathrm{H}, 2^{\prime}-\mathrm{H}$ T5), 2.29 (ddd partially overlapping with previous signal, $\mathrm{J}_{2^{\prime \prime}, 2^{\prime}}=-14.1, \mathrm{~J}_{2^{\prime \prime}, 1^{\prime}}=6.9, \mathrm{~J}_{2^{\prime \prime}, 3^{\prime}}=4.5 \mathrm{~Hz}, 1 \mathrm{H}, 2^{\prime \prime}-\mathrm{H}$ T5), $2.48-2.55$ (partially overlapped multiplets, $3 \mathrm{H}, 2^{\prime}-\mathrm{H}$ A1, A2 and A3), $2.61\left(\mathrm{dd}, \mathrm{J}_{2^{\prime \prime}, 2^{\prime}}=-13.8, \mathrm{~J}_{2^{\prime \prime}, 1^{\prime}}=5.0, \mathrm{~J}_{2^{\prime \prime}, 3^{\prime}}=0 \mathrm{~Hz}, 1 \mathrm{H}, 2^{\prime \prime}-\mathrm{H}\right.$ A3), 2.65-2.77 (partially overlapped multiplets, 4 H, 2'-H A4, 2'-H A1, A2 and A4), 3.49 (dd, Ja, $\mathrm{J}_{1 \mathrm{~b}}=-11.8$, $\mathrm{J}_{1 \mathrm{a}, 2}=6.2 \mathrm{~Hz}, 1 \mathrm{H}, 1 \mathrm{a}-\mathrm{H}$ of glycerol), $3.58\left(\mathrm{dd}, \mathrm{J}_{\mathrm{bb}, 1 \mathrm{a}}=-11.8, \mathrm{~J}_{\mathrm{bb}, 2}=4.2 \mathrm{~Hz}, 1 \mathrm{H}, 1 \mathrm{~b}-\mathrm{H}\right.$ of glycerol), $3.71(\mathrm{~m}, 1$ $\mathrm{H}, 3 \mathrm{a}-\mathrm{H}$ of glycerol), 3.81 (overlapped multiplets, $2 \mathrm{H}, 2-\mathrm{H}$ and $3 \mathrm{~b}-\mathrm{H}$ of glycerol), 3.96 [ddd, $\mathrm{J}_{5}, 5^{\prime \prime}=-11.7$, $\Sigma\left(\mathrm{J}_{5^{\prime}, 4^{\prime}}+\mathrm{J}_{5^{\prime}, \mathrm{P}}\right)=7.3 \mathrm{~Hz}, 1 \mathrm{H}, 5^{\prime}-\mathrm{H}$ A1], $4.05\left(\mathrm{dt}, \mathrm{J}_{5^{\prime \prime}, 5^{\prime}}=-11.7, \mathrm{~J}_{5^{\prime \prime}, 4^{\prime}}=\mathrm{J}_{5^{\prime \prime}, \mathrm{P}}=4.0 \mathrm{~Hz}, 1 \mathrm{H}, 5^{\prime \prime}-\mathrm{H} \mathrm{A1}\right), 4.10-4.26$ (partially overlapped multiplets, $9 \mathrm{H}, 4^{\prime}-\mathrm{H}$ T5, 5'- $\mathrm{H}_{2}$ T5, A2, A3 and A4), 4.37-4.40 (partially overlapped multiplets, 3 H, 4'-H A1, A2 and A3), 4.44 (m, 1 H, 4'-H A4), 4.57 (ddd, $\mathrm{J}_{3^{\prime}, 2^{\prime}}=6.8, \mathrm{~J}_{3^{\prime}, 2^{\prime}}=4.5, \mathrm{~J}_{3^{\prime}, 4^{\prime}}=3.8 \mathrm{~Hz}$, 1 H, 3'-H T5), 4.97 (m, 1 H, 3'-H A1), 5.01 (overlapped multiplets, 3 H, 3'-H A2, A3 and A4), 5.80 (dd, J ${ }^{\prime}, 2^{\prime}=$ 9.6, $\left.\mathrm{J}_{1^{\prime}, 2^{\prime \prime}}=5.0 \mathrm{~Hz}, 1 \mathrm{H}, 1^{\prime}-\mathrm{H} \mathrm{A} 3\right), 5.90\left(\mathrm{dd}, \mathrm{J}_{1^{\prime}, 2^{\prime}}=9.3, \mathrm{~J}_{1^{\prime} 2^{\prime \prime}}=5.2 \mathrm{~Hz}, 1 \mathrm{H}, 1^{\prime}-\mathrm{H} \mathrm{A} 2\right), 6.07\left(\mathrm{dd}, \mathrm{J}_{1^{\prime}, 2^{\prime}}=8.3, \mathrm{~J}_{1^{\prime}, 2^{\prime \prime}}\right.$ $=6.0 \mathrm{~Hz}, 1 \mathrm{H}, 1^{\prime}-\mathrm{H} \mathrm{A}$ ) $), 6.12$ (t, J = $\left.6.9 \mathrm{~Hz}, 1 \mathrm{H}, 1^{\prime}-\mathrm{H} \mathrm{T} 5\right), 6.20\left(\mathrm{dd}, \mathrm{J}_{1^{\prime}, 2^{\prime}}=7.7, \mathrm{~J}_{1^{\prime}, 2^{\prime}}=6.3 \mathrm{~Hz}, 1 \mathrm{H}, 1^{\prime}-\mathrm{H} \mathrm{A} 4\right)$, 7.44 (br. q, J 0.9 Hz, 1 H, 6-H T5), 7.72 (br. s, 2 H, 2-H A2 and A3), 7.92 (s, 1 H, 2-H A1), 8.04 (s, 1 H, 2-H A4), 8.17 (s, 1 H, 8-H A1), 8.19 (s, 1 H, 8-H A2), 8.25 (s, 1 H, 8-H A3), 8.33 (s, 1 H, 8-H A4) ppm.

${ }^{13} \mathrm{C}_{\mathrm{NMR}}\left(\mathrm{D}_{2} \mathrm{O}\right): \delta 14.3\left(\mathrm{CH}_{3} \mathrm{~T}\right.$ T), 39.7 (C-2' A3), 40.0 (C-2' A2), 40.2 (C-2’ A1), 40.8 (C-2' A4), 41.5 (C2' T5), 64.9 (C-1 of glycerol), 67.1 (d, J $\mathrm{JOP}_{\mathrm{COP}}=3.6 \mathrm{~Hz}, \mathrm{C}-5$ ' T5), 67.6 (d, J $\mathrm{J}_{\mathrm{COP}}=3.9 \mathrm{~Hz}, \mathrm{C}-5$ ' A4), 68.0 (br. d, $\mathrm{J}_{\mathrm{COP}} \sim 5.0 \mathrm{~Hz}, \mathrm{C}-5^{\prime}$ A2 and A3), $68.2\left(\mathrm{~d}, \mathrm{~J}_{\mathrm{COP}}=5.1 \mathrm{~Hz}, \mathrm{C}-5^{\prime}\right.$ A1), $69.2\left(\mathrm{~d}, \mathrm{~J}_{\mathrm{COP}}=5.5 \mathrm{~Hz}, \mathrm{C}-3\right.$ of glycerol), 72.4 (C-3’ T5), 73.5 (d, J $\mathrm{CCOP}=7.9 \mathrm{~Hz}, \mathrm{C}-2$ of glycerol), 78.1 (d, J $\mathrm{COP}=5.3 \mathrm{~Hz}, \mathrm{C}-3^{\prime}$ A), 79.1 (d, J $\mathrm{JOP}_{\mathrm{COP}}=5.2 \mathrm{~Hz}, \mathrm{C}-3^{\prime}$ A), $79.3\left(\mathrm{~d}, \mathrm{~J}_{\mathrm{COP}}=4.6 \mathrm{~Hz}, \mathrm{C}-3^{\prime}\right.$ A), $80.0\left(\mathrm{~d}, \mathrm{~J}_{\mathrm{COP}}=5.4 \mathrm{~Hz}, \mathrm{C}-3^{\prime}\right.$ A1), 84.8 (C-1' A3), 84.9 (C-1' A2), 85.1 (C1' A4), 86.1 (C-1' A1), 86.9 (C-1' T5), 87.1 (t, J $\mathrm{JCOP}_{\mathrm{COP}}=9.8 \mathrm{~Hz}, \mathrm{C}-4$ ' A4), 87.3 (d, J $\mathrm{CCOP}=8.7 \mathrm{~Hz}, \mathrm{C}-4$ ' T5), $87.5\left(t, J_{\mathrm{CCOP}}=9.4 \mathrm{~Hz}, \mathrm{C}-4{ }^{\prime} \mathrm{A} 2\right.$ and A3), $88.0\left(\mathrm{t}, \mathrm{J}_{\mathrm{CCOP}}=9.4 \mathrm{~Hz}, \mathrm{C}-4{ }^{\prime} \mathrm{A} 1\right), 114.0(\mathrm{C}-5$ T5), 119.8, 120.0, 120.3 and 121.0 (C-5 A1, A2, A3 and A4), 139.5 (C-6 T5), 141.7 (C-8 A4 and A3), 142.0 (C-8 A2), 142.2 (C-8 A1), 
150.1, 150.2, 150.5 and 150.9 (C-4 A1, A2, A3 and A4), 153.8 (C-2 T5), 154.7 and 154.8 (C-2 A2, and A3), 155.0 (C-2 A1 and A4), 156.9, 157.0, 157.4 and 157.8 (C-6 A1, A2, A3 and A4), 168.4 (C-4 T5) ppm. 
[5'-GPd(ApTpApTpTpA) (6)]. ${ }^{1} \mathrm{H}$ NMR (D $\left(\mathrm{D}_{2} \mathrm{O}\right): \delta 1.73$ (br. d, J $\left.\sim 0.9 \mathrm{~Hz}, 3 \mathrm{H}, \mathrm{CH}_{3} \mathrm{~T} 2\right), 1.75$ (br. d, J $~ 0.9$ $\mathrm{Hz}, 3 \mathrm{H}, \mathrm{CH}_{3} \mathrm{~T} 4$ ), 1.86 (br. d, J $~ 0.9 \mathrm{~Hz}, 3 \mathrm{H}, \mathrm{CH}_{3} \mathrm{~T} 5$ ), 1.85-1.92 (partially overlapped multiplets $2 \mathrm{H}, 2^{\prime}-\mathrm{H}$ T5 and T2), 2.22-2.32 (partially overlapped multiplets, 3 H, 2'-H T4, 2'-H T2 and T5), 2.46 (ddd, $\mathrm{J}_{2,2}{ }^{\prime}=-$ $14.2, \mathbf{J}_{2^{\prime \prime}, 1^{\prime}}=6.0, \mathbf{J}_{2^{\prime \prime}, 3^{\prime}}=2.1 \mathrm{~Hz}, 1 \mathrm{H}, 2^{\prime \prime}-\mathrm{H}$ T4), $2.63\left(\mathrm{ddd}, \mathrm{J}_{2^{\prime \prime}, 2^{\prime}}=-13.9, \mathrm{~J}_{2^{\prime}, 1^{\prime}}=6.7, \mathbf{J}_{2^{\prime \prime}, 3^{\prime}}=4.2 \mathrm{~Hz}, 1 \mathrm{H}, 2^{\prime \prime}-\mathrm{H}\right.$ A6), 2.80-2.83 (partially overlapped multiplets, 4 H, 2'-H and 2"-H A1, 2'-H and 2"'-H A3), 2.88 (ddd, J', $2^{\prime \prime}=$ $\left.-13.9, \mathrm{~J}_{2^{\prime}, 1^{\prime}}=6.7, \mathrm{~J}_{2^{\prime}, 3^{\prime}}=6.6 \mathrm{~Hz}, 1 \mathrm{H}, 2^{\prime}-\mathrm{H} \mathrm{A6}\right), 3.50\left(\mathrm{dd}, \mathrm{J}_{1 \mathrm{a}, 1 \mathrm{~b}}=-11.8, \mathrm{~J}_{1 \mathrm{a}, 2}=6.3 \mathrm{~Hz}, 1 \mathrm{H}, 1 \mathrm{a}-\mathrm{H}\right.$ of glycerol $)$, $3.59\left(\mathrm{dd}, \mathrm{J}_{1 \mathrm{~b}, 1 \mathrm{a}}=-11.8, \mathrm{~J}_{1 \mathrm{~b}, 2}=4.4 \mathrm{~Hz}, 1 \mathrm{~b}-\mathrm{H}\right.$ of glycerol), $3.73(\mathrm{~m}, 1 \mathrm{H}, 3 \mathrm{a}-\mathrm{H}$ of glycerol), 3.83 (overlapped multiplets, $2 \mathrm{H}, 2-\mathrm{H}$ and 3b-H of glycerol), 4.03 (m, 1 H, 5'-H A6), 4.07-4.20 (partially overlapped multiplets, $12 \mathrm{H}, 5$ '-H and 5"'-H A1, T2, A3, T4, T5, 4'-H and 5"'-H A6), 4.25 and 4.27 (partially overlapped multiplets, 2 H altogether, 4'-H T2 and T5, respectively), 4.32 (m, 1 H, 4'-H T4), 4.45 and 4.47 (partially overlapped multiplets, $2 \mathrm{H}$ altogether, 4'-H A3 and A1, respectively), 4.78-4.86 (overlapped multiplets partially obscured by residual HOD signal, 3 H, 3'-H T2, T5 and A6), 4.89 (m, 1 H, 3'-H T4), 5.03 (overlapped multiplets, 2 H, 3'-H A1 and A3), $6.02\left(\mathrm{dd}, \mathrm{J}_{1^{\prime}, 2^{\prime}}=8.8, \mathrm{~J}_{1^{\prime}, 2^{\prime \prime}}=5.9 \mathrm{~Hz}, 1 \mathrm{H}, 1^{\prime}-\mathrm{H}\right.$ T2), $6.06\left(\mathrm{dd}^{\prime} \mathrm{J}_{1^{\prime}, 2^{\prime}}=8.6, \mathrm{~J}_{1^{\prime}, 2^{\prime}}=6.0 \mathrm{~Hz}, 1 \mathrm{H}\right.$, $1^{\prime}-\mathrm{H}$ T5), $6.16\left(\mathrm{dd}, \mathrm{J}_{1^{\prime}, 2^{\prime}}=8.2, \mathrm{~J}_{1^{\prime}, 2^{\prime}}=6.0 \mathrm{~Hz}, 1 \mathrm{H}, 1^{\prime}-\mathrm{H} \mathrm{T} 4\right), 6.31\left(\mathrm{dd}, \mathrm{J}_{1^{\prime}, 2^{\prime}}=7.3, \mathrm{~J}_{1^{\prime}, 2^{\prime}}=6.7 \mathrm{~Hz}, 1 \mathrm{H}, 1^{\prime}-\mathrm{H} \mathrm{A} 3\right)$, $6.38\left(\mathrm{dd}, \mathrm{J}_{1^{\prime}, 2^{\prime}}=7.1, \mathrm{~J}_{1^{\prime}, 2^{\prime}}=6.9 \mathrm{~Hz}, 1 \mathrm{H}, 1^{\prime}-\mathrm{H} \mathrm{A1}\right), 6.43\left(\mathrm{t}, \mathrm{J}=6.7 \mathrm{~Hz}, 1 \mathrm{H}, 1^{\prime}-\mathrm{H}\right.$ A6), 7.35 (br. q, J 0.9 Hz, 1 H, 6-H T2), 7.45 (br. q, J 0.9 Hz, 1 H, 6-H T5), 7.53 (br. q, J 0.9 Hz, 1 H, 6-H T4), 8.07, 8.17 and 8.19 (singlets, $3 \mathrm{H}$ altogether, 2-H A1, A3 and A6), 8.40 and 8.41 (partially overlapped singlets, $1 \mathrm{H}$ and $2 \mathrm{H}$ respectively, 8-H A1, A3 and A6) ppm. 
[5'-GPd(GpA) (7)]. ${ }^{1} \mathrm{H}$ NMR ( $\left.\mathrm{D}_{2} \mathrm{O}\right): \delta 2.40\left(\mathrm{ddd}, \mathrm{J}_{2^{\prime}, 2^{\prime \prime}}=-14.2, \mathrm{~J}_{2^{\prime}, 1^{\prime}}=9.1, \mathrm{~J}_{2^{\prime}, 3^{\prime}}=5.4 \mathrm{~Hz}, 1 \mathrm{H}, 2^{\prime}-\mathrm{H} \mathrm{G} 1\right), 2.51$ $\left(\mathrm{ddd}, \mathrm{J}_{2^{\prime \prime}, 2^{\prime}}=-13.9, \mathrm{~J}_{2^{\prime}, 1^{\prime}}=6.4, \mathbf{J}_{2^{\prime \prime}, 3^{\prime}}=4.0 \mathrm{~Hz}, 1 \mathrm{H}, 2^{\prime \prime}-\mathrm{H} \mathrm{A} 2\right), 2.66\left(\mathrm{dd}, \mathrm{J}_{2^{\prime \prime}, 2^{\prime}}=-14.2, \mathrm{~J}_{2^{\prime}, 1^{\prime}}=5.5, \mathrm{~J}_{2^{\prime \prime}, 3^{\prime}}=0 \mathrm{~Hz}, 1 \mathrm{H}\right.$, 2"-H G1), $2.75\left(\mathrm{ddd}, \mathrm{J}_{2^{\prime}, 2^{\prime \prime}}=-13.9, \mathrm{~J}_{2}, \mathrm{l}^{\prime}=7.2, \mathrm{~J}_{2^{\prime}, 3^{\prime}}=6.8 \mathrm{~Hz}, 1 \mathrm{H}, 2^{\prime}-\mathrm{H} \mathrm{A} 2\right), 3.53\left(\mathrm{dd}, \mathrm{J}_{\mathrm{la}, 1 \mathrm{~b}}=-11.7, \mathrm{~J}_{\mathrm{la}, 2}=6.0 \mathrm{~Hz}, 1\right.$ $\mathrm{H}, 1 \mathrm{a}-\mathrm{H}$ of glycerol), $3.61\left(\mathrm{dd}, \mathrm{J}_{1 \mathrm{~b}, 1 \mathrm{a}}=-11.7, \mathrm{~J}_{1 \mathrm{~b}, 2}=4.2 \mathrm{~Hz}, 1 \mathrm{H}, 1 \mathrm{~b}-\mathrm{H}\right.$ of glycerol), $3.73(\mathrm{~m}, 1 \mathrm{H}, 3 \mathrm{a}-\mathrm{H}$ of glycerol), 3.79-3.86 (overlapped multiplets, 2 H, 2-H and 3b-H of glycerol), 3.94 (m, 1 H, 5'-H G1), 4.03 (m, 1 H, 5'”-H G1), 4.11-4.22 (partially overlapped multiplets, 3 H, 4'-H, 5'-H and 5' -H A2), 4.34 (m, 1 H, 4'-H G1), 4.77 (ddd, $\mathrm{J}_{3^{\prime}, 2^{\prime}}=6.8, \mathrm{~J}_{3^{\prime}, 2^{\prime}}=4.0, \mathrm{~J}_{3^{\prime}, 4^{\prime}}=3.8 \mathrm{~Hz}, 1 \mathrm{H}, 3^{\prime}-\mathrm{H}$ A2), 4.80 (m partially obscured by the residual HOD signal, 3'-H G1), $6.06\left(\mathrm{dd}, \mathrm{J}_{1^{\prime}, 2^{\prime}}=7.2, \mathrm{~J}_{1^{\prime}, 2^{\prime}}=6.4 \mathrm{~Hz}, 1 \mathrm{H}, 1^{\prime}-\mathrm{H} \mathrm{A} 2\right), 6.19\left(\mathrm{dd}, \mathrm{J}_{1^{\prime}, 2^{\prime}}=9.1, \mathrm{~J}_{1^{\prime}, 2^{\prime}}=5.5 \mathrm{~Hz}, 1 \mathrm{H}, 1^{\prime}-\mathrm{H} \mathrm{G1}\right), 7.91(\mathrm{~s}$, 1 H, 8-H G1), 8.08 (s, 1 H, 2-H A2), 8.11 (s, 1 H, 8-H A2) ppm. 
[5'-GPd(TpA) (8)]. ${ }^{1} \mathrm{H}$ NMR $\left(\mathrm{D}_{2} \mathrm{O}\right): \delta 1.88\left(\mathrm{ddd}, \mathrm{J}_{2^{\prime}, 2^{\prime}}=-14.3, \mathrm{~J}_{2^{\prime}, 1^{\prime}}=8.8, \mathrm{~J}_{2^{\prime}, 3^{\prime}}=5.5 \mathrm{~Hz}, 1 \mathrm{H}, 2^{\prime}-\mathrm{H} \mathrm{T1}\right), 1.95(\mathrm{~d}$, $\left.\mathrm{J}=1.0 \mathrm{~Hz}, 3 \mathrm{H}, \mathrm{CH}_{3} \mathrm{~T} 1\right), 2.28\left(\mathrm{dd}, \mathrm{J}_{2^{\prime \prime}, 2^{\prime}}=-14.3, \mathrm{~J}_{2^{\prime \prime}, 1^{\prime}}=5.8, \mathrm{~J}_{2^{\prime \prime}, 3^{\prime}}=0 \mathrm{~Hz}, 1 \mathrm{H}, 2^{\prime \prime}-\mathrm{H} \mathrm{T1}\right), 2.65\left(\mathrm{ddd}, \mathrm{J}_{2^{\prime \prime}, 2^{\prime}}=-14.3\right.$, $\left.\mathrm{J}_{2^{\prime}, 1^{\prime}}=6.7, \mathrm{~J}_{2^{\prime}, 3^{\prime}}=4.2 \mathrm{~Hz}, 1 \mathrm{H}, 2^{\prime}{ }^{\prime}-\mathrm{H} \mathrm{A} 2\right), 2.94\left(\mathrm{ddd}, \mathrm{J}_{2^{\prime}, 2^{\prime}}=-14.3, \mathrm{~J}_{2^{\prime}, 1^{\prime}}=6.7, \mathrm{~J}_{2^{\prime}, 3^{\prime}}=6.4 \mathrm{~Hz}, 1 \mathrm{H}, 2^{\prime}-\mathrm{H} \mathrm{A} 2\right), 3.63$ $\left(\mathrm{dd}, \mathrm{J}_{1 \mathrm{a}, 1 \mathrm{~b}}=-12.0, \mathrm{~J}_{1 \mathrm{a}, 2}=6.0 \mathrm{~Hz}, 1 \mathrm{H}, 1 \mathrm{a}-\mathrm{H}\right.$ of glycerol), $3.70\left(\mathrm{dd}, \mathrm{J}_{\mathrm{bb}, 1 \mathrm{a}}=-12.0, \mathrm{~J}_{1 \mathrm{~b}, 2}=4.0 \mathrm{~Hz}, 1 \mathrm{H}, 1 \mathrm{~b}-\mathrm{H}\right.$ of glycerol), 3.88 (m, $1 \mathrm{H}, 3 \mathrm{a}-\mathrm{H}$ of glycerol), 3.91-3.96 (overlapped multiplets, $2 \mathrm{H}, 2-\mathrm{H}$ and 3b-H of glycerol), 3.98 (ddd partially overlapped with the next signal, $\mathrm{J}_{5^{\prime \prime}, 5^{\prime}}=-11.6, \mathrm{~J}_{5^{\prime \prime}, 4^{\prime}}=3.3, \mathrm{~J}_{5^{\prime}, \mathrm{P}}=4.0 \mathrm{~Hz}, 1 \mathrm{H}, 5^{\prime \prime}-\mathrm{H} \mathrm{A} 2$ ), 4.02 (ddd partially overlapped with the previous signal, $\left.\mathrm{J}_{5^{\prime}, 5^{\prime \prime}}=-11.6, \mathrm{~J}_{5^{\prime}, 4^{\prime}}=3.9, \mathrm{~J}_{5^{\prime}, \mathrm{P}}=4.3 \mathrm{~Hz}, 1 \mathrm{H}, 5^{\prime}-\mathrm{H} \mathrm{A} 2\right), 4.14\left(\mathrm{t}, \mathrm{J}_{5^{\prime}, 4^{\prime}}=\right.$ 3.0, J ${ }^{\prime}, \mathrm{P}=3.0 \mathrm{~Hz}, 2 \mathrm{H}, 5^{\prime}-\mathrm{H} \mathrm{T1}$ ), 4.23 (m, 1 H, 4'-H A2), 4.29 (m, 1 H, 4'-H T1), 4.84 (m, 1 H, 3'-H T1), 6.10(dd, $\left.\mathrm{J}_{1^{\prime}, 2^{\prime}}=8.8, \mathrm{~J}_{1^{\prime}, 2^{\prime \prime}}=5.8 \mathrm{~Hz}, 1 \mathrm{H}, 1^{\prime}-\mathrm{H} \mathrm{T1}\right), 6.49\left(\mathrm{t}, \mathrm{J}_{1^{\prime}, 2^{\prime}}=\mathrm{J}_{1^{\prime}, 2^{\prime \prime}}=6.7 \mathrm{~Hz}, 1 \mathrm{H}, 1^{\prime}-\mathrm{H} \mathrm{A} 2\right), 7.48(\mathrm{q}, \mathrm{J}=1.0 \mathrm{~Hz}, 1 \mathrm{H}, 6-\mathrm{H}$ T1), 8.25 (s, $1 \mathrm{H}, 2-\mathrm{H} \mathrm{A} 2), 8.48$ (s, $1 \mathrm{H}, 8-\mathrm{H} \mathrm{A}$ ) ppm. The 3'-H A2 resonance was obscured by the residual HOD signal. 
[1-O-Benzoyl-3- $\boldsymbol{O}$-(4,4'-dimethoxytrityl)-sn-glycerol (16) ]. ${ }^{1} \mathrm{H}$ NMR $\left(\mathrm{CDCl}_{3}\right)$ : glycerol moiety, $\delta 3.33(\mathrm{~m}$, $\left.2 \mathrm{H}, 3-\mathrm{H}_{2}\right), 4.13(\mathrm{~m}, 1 \mathrm{H}, 2-\mathrm{H}), 4.43\left(\mathrm{dd}, \mathrm{J}_{1 \mathrm{a}, 1 \mathrm{~b}}=-11.5, \mathrm{~J}_{1 \mathrm{a}, 2}=5.0 \mathrm{~Hz}, 1 \mathrm{H}, 1 \mathrm{a}-\mathrm{H}\right), 4.46\left(\mathrm{dd}, \mathrm{J}_{1 \mathrm{~b}, 1 \mathrm{a}}=-11.5, \mathrm{~J}_{1 \mathrm{~b}, 2}=\right.$ $4.0 \mathrm{~Hz}, 1 \mathrm{H}, 1 \mathrm{~b}-\mathrm{H}$ ); benzoyl moiety, $\delta 7.43(\mathrm{t}, \mathrm{J}=7.5 \mathrm{~Hz}, 2 \mathrm{H}, 3-\mathrm{H}$ and $5-\mathrm{H}), 7.57$ (tt, $\mathrm{J}_{\text {ortho }}=7.5, \mathrm{~J}_{\text {meta }}=1.0 \mathrm{~Hz}$, $1 \mathrm{H}, 4-\mathrm{H}), 7.96\left(\mathrm{dd}, \mathrm{J}_{\text {ortho }}=7.5, \mathrm{~J}_{\text {meta }}=1.0 \mathrm{~Hz}, 2 \mathrm{H}, 2-\mathrm{H}\right.$ and 6-H); methoxyphenyl moieties, $\delta 3.77(\mathrm{~s}, 6 \mathrm{H}$, $\left.\mathrm{OCH}_{3}\right), 6.81(\mathrm{~d}, \mathrm{~J}=8.0 \mathrm{~Hz}, 4 \mathrm{H}, 3-\mathrm{H}$ and 5-H), $7.33(\mathrm{~d}, \mathrm{~J}=8.0 \mathrm{~Hz}, 4 \mathrm{H}, 2-\mathrm{H}$ and 6-H); phenyl moiety, $\delta$ 7.21 $\left(\mathrm{tt}, \mathrm{J}_{\text {ortho }}=7.5, \mathrm{~J}_{\text {meta }}=1.0 \mathrm{~Hz}, 1 \mathrm{H}, 4-\mathrm{H}\right) 7.28(\mathrm{t}, \mathrm{J}=7.5 \mathrm{~Hz}, 2 \mathrm{H}, 3-\mathrm{H}$ and $5-\mathrm{H}), 7.44\left(\mathrm{dd}, \mathrm{J}_{\text {ortho }}=7.5, \mathrm{~J}_{\text {meta }}=1.0\right.$ $\mathrm{Hz}, 2 \mathrm{H}, 2-\mathrm{H}$ and 6-H) ppm. ${ }^{13} \mathrm{C}$ NMR (CDCl $)$ : glycerol moiety, $\delta 64.6$ (C-3), 66.8 (C-1), 70.1 (C-2); benzoyl moiety, $\delta 129.1$ (C-3 and C-5), 130.4 (C-2 and C-6), 130.5 (C-1), 133.9 (C-4), 167.3 (CO); methoxyphenyl moieties, $\delta 55.9\left(\mathrm{OCH}_{3}\right), 113.9(\mathrm{C}-3$ and C-5), 130.7 (C-2 and C-6), $136.5(\mathrm{C}-1), 159.3$ (C-4); phenyl moiety, $\delta$ 127.6 (C-4), 128.6 (C-2 and C-6), 128.8 (C-3 and C-5), $145.4(\mathrm{C}-1) ; 87.0\left(\mathrm{Ar}_{3} \mathrm{CO}\right) \mathrm{ppm}$. 